\title{
Supramolecular Platform Stabilizing Growth Factors
}

\author{
Simone I. S. Hendrikse, Sergio Spaans, E. W. Meijer,*(i) and Patricia Y. W. Dankers*(1) \\ Institute for Complex Molecular Systems, Eindhoven University of Technology, P.O. Box 513, 5600 MB Eindhoven, The Netherlands
}

\section{Supporting Information}

ABSTRACT: High concentrations of supplemented growth factors can cause oversaturation and adverse effects in in vitro and in vivo studies, though these supraphysiological concentrations are often required due to the low stability of growth factors. Here we demonstrate the stabilization of TGF- $\beta 1$ and BMP4 using supramolecular polymers. Inspired by heparan sulfate, sulfonated peptides were presented on a supramolecular polymer to allow for noncovalent binding to growth factors in solution. After mixing with excipient molecules, both TGF- $\beta 1$ and BMP4 were shown to have a prolonged half-life compared to the growth factors free in solution. Moreover, high cellular response was measured by a luciferase assay, indicating that TGF- $\beta 1$ remained highly active upon binding to the supramolecular assembly. The results demonstrate that significant lower concentrations of growth factors can be used when supramolecular polymers bearing growth factor binding moieties are implemented. This approach can also be exploited in hydrogel systems to control growth factor release.

\section{INTRODUCTION}

Growth factors are involved in many cellular processes such as cell survival, proliferation, differentiation, and migration. ${ }^{1,2}$ In the extracellular matrix (ECM), sulfated glycosaminoglycans (GAGs) present on proteoglycans are known to bind various heparan sulfate/heparin-binding growth factors that mediate cellular delivery and subsequent intracellular signaling. ${ }^{3}$ The negatively charged sulfates and carboxylic acids present on, for example, heparan sulfate interact via electrostatic interactions with positively charged regions, which are rich in lysine and arginine, within the growth factor. ${ }^{4}$ Depending on the growth factor, hydrogen bonding and hydrophobic effects can have a significant contribution as well. ${ }^{5}$ Immobilization of growth factors to heparan sulfate results in protection from degradation and inactivation. Moreover, the local high concentration as a result of this binding allows for multivalent interactions, contributing to spatiotemporal presentation. In contrast, for in vitro and in vivo studies, growth factors are typically administrated in supraphysiological concentrations due to a lack of controlled delivery, slow diffusion, and low stability over time, leading to suboptimal conditions and possibly adverse effects. ${ }^{6}$ In addition, growth factors are usually diluted in high concentrations of bovine serum albumin (BSA) to enhance the half-life. However, BSA binds to several other components as well and is known to influence both metabolic and biosynthetic processes in cells. ${ }^{7}$ Therefore, there is an increasing need to develop scaffolds capable of binding growth factors, maintaining their activity, and presenting the growth factors to the cell in a spatiotemporal manner.

To improve the efficiency of growth factor presentation compared to soluble supplementation, growth factors can be immobilized by either physical entrapment in a hydrogel system $^{8}$ or by noncovalent ${ }^{9}$ or covalent ${ }^{10}$ conjugation. ${ }^{11}$ To elucidate the role of participating functional groups in protein binding and to investigate the sustained release of growth factors, heparin-based systems, ${ }^{12}$ polymers, ${ }^{13}$ monosaccharides, ${ }^{14}$ synthetic peptides, ${ }^{15,16}$ multicomponent assemblies, ${ }^{17}$ and molecules ${ }^{18}$ have been developed that have different degrees of sulfates and sulfonates. Moreover, synthetic peptides that specifically target certain growth factors are also established. ${ }^{19}$ Although successful conjugation strategies have been developed to covalently attach growth factors to polymers, ${ }^{10}$ care must be taken considering the fact that conformational changes and inactivation are likely to occur. Another consideration to take into account is that growth factor receptor presentation might be hampered when the binding is too strong or encapsulation too efficient. Therefore, tuning the number of noncovalent interactions might be beneficial to enhance both growth factor stabilization and cell receptor binding.

Supramolecular polymers are proposed to serve as excellent ECM mimics since their self-assembly of monomers, which is driven by noncovalent interactions, gives rise to fibrous structures with an inherent dynamic nature closely resembling ECM properties. ${ }^{20}$ Because of their tunability, functional monomers can be simply coassembled with scaffolding monomers to arrive at multicomponent functional biomaterials. Supramolecular polymers based on peptide amphiphiles have previously been shown to bind the growth factor TGF- $\beta 1$ by a short peptide sequence (HSNGLPL) ${ }^{21}$ and several other growth factors by a sulfated monosaccharide. ${ }^{14}$ Inspired by heparan sulfate, which is able to bind different growth factors

Received: February 8, 2018

Revised: April 15, 2018

Published: April 20, 2018 
by distinct binding affinities, ${ }^{5}$ the short synthetic tetrapeptide discovered by Maynard and Hubbell ${ }^{22}$ also serves as an attractive candidate. This peptide displays the three functional groups of heparan sulfate and heparin, that is, sulfates, carboxylates, and hydroxyl groups, and can be easily synthesized and incorporated into artificial systems. Moreover, they showed the importance of a carboxylate between the two sulfated tyrosines compared to a solely negatively charged peptide on the binding to vascular endothelial growth factor (VEGF). In addition, Kim and Kiick investigated the influence of more sulfated tyrosines included in this peptide and confirmed that this shorter peptide has the highest binding affinity to VEGF and heparin binding peptides due to a reduced steric hindrance or repulsion upon binding. ${ }^{23}$

Rather than investigating the sustained release of growth factors from hydrogels, we here sought to elucidate the stabilization effects of excipient molecules in the diluted state using self-assembled ureidopyrimidinone (UPy) based supramolecular polymers. The self-complementary UPy selfassembles into fibrillar 1D fibers by first dimerization due to quadruple hydrogen bonding and subsequent lateral stacking guided by both hydrophobic effects and hydrogen bonding between the urea groups. ${ }^{24}$ To equip the supramolecular polymers with growth factor binding sites, UPy polymers were coassembled with a sulfonated peptide to prolong the stability of transforming growth factor- $\beta 1$ (TGF- $\beta 1$ ) and bone morphogenic protein-4 (BMP4). The stability of both growth factors was assessed at $37{ }^{\circ} \mathrm{C}$ with different scaffolds (i.e., excipients) in solution. Upon incorporation of sulfonated peptides in a supramolecular fiber, noncovalent protein interactions enhance growth factor stability over physical adsorption. As a proof-of-principle, cell experiments were carried out with TGF- $\beta 1$ sensitive cells to assess whether the stabilized TGF- $\beta 1$ was still able to induce a cellular response, that is, the conversion of active TGF- $\beta 1$ into luciferase.

\section{EXPERIMENTAL SECTION}

Materials. Chemicals and reagents were purchased from Sigma and Novabiochem and used as received unless otherwise indicated. (S)Fmoc-phenylalanine-4-sulfonic acid was purchased from PepTech Corporation. Peptides and conjugates were purified on a $\mathrm{C} 18$ automated column with a gradient of 5 to $100 \%$ acetonitrile in water using a Buchi Reveleris system. Recombinant human TGF- $\beta 1$ (HEK293 derived) was purchased from Peprotech and Recombinant human BMP-4 (carrier free) from R\&D systems. Enzyme-linked immunosorbent assays (ELISA) were performed using human TGF$\beta 1$ Quantikine ELISA kits or human BMP-4 Quantikine ELISA kits from R\&D systems. Luciferase assay kit was purchased from Promega. Phosphate buffered saline (PBS) tablets were purchased from SigmaAldrich ( $\mathrm{pH} 7.20-7.60)$. Trypsin-EDTA solution was purchased from Sigma $(0.5 \mathrm{~g} / \mathrm{L}$ porcine trypsin and $0.2 \mathrm{~g} / \mathrm{L}$ EDTA in Hank's Balanced Salt Solution with phenol red).

Methods. Analytical Techniques. Reversed-phase high performance liquid chromatography-mass spectrometry (RP-HPLC-MS) was performed using a Shimadzu instrument. The amount of active TGF- $\beta 1$ bound to the ELISA kit was assessed by measuring the absorbance at $450 \mathrm{~nm}$, with a wavelength correction set to $570 \mathrm{~nm}$, using a microplate reader from Safire II. Ultraviolet-visible (UV-vis) spectrophotometry was performed on a Jasco V-650 instrument. The relative amount of luciferase expressed by cells was evaluated by measuring the luminescence intensity using a microplate reader from Synergy HT.

Reconstitution of TGF- $\beta 1$ and BMP4. TGF- $\beta 1$ and BMP4 were reconstituted according to manufacturer's protocol. Briefly, TGF- $\beta 1$ was dissolved in $10 \mathrm{mM}$ citric acid, whereas BMP4 was dissolved in 4 $\mathrm{mM} \mathrm{HCl}$. After $30 \mathrm{~min}$, the growth factors were aliquoted in $0.1 \%$ BSA in PBS, or PBS (BSA free) at a $5 \mu \mathrm{g} / \mathrm{mL}$ concentration and stored at $-20{ }^{\circ} \mathrm{C}$ before usage.

Synthesis of Sulfated Tyrosine for SP1. Fmoc-Tyr $(\mathrm{tBu})-\mathrm{OH}(4.35$ $\mathrm{mmol}$ ) was deprotected in a cleavage cocktail containing TFA, TIS, and water (95:2.5:2.5) for $2 \mathrm{~h}$ at room temperature. Subsequently, the TFA was partly evaporated, precipitated in cold hexane/ether (1:1), incubated for $15 \mathrm{~min}$ at $-20^{\circ} \mathrm{C}$, and centrifuged for $10 \mathrm{~min}$ at $20 \mathrm{k}$ RPM. The supernatant was removed and the pellet was redissolved in water/acetonitrile, and after lyophilization a white solid was obtained in $91.1 \%$ yield $(1.60 \mathrm{~g}) . \mathrm{LC}-\mathrm{MS}: \mathrm{M} w_{\text {calc }}=403.43 \mathrm{~g} / \mathrm{mol}, \mathrm{m} / z_{\mathrm{obs}}=$ $404.08[\mathrm{M}+\mathrm{H}]^{+}$. Subsequently, Fmoc-Tyr $(\mathrm{OH})-\mathrm{OH}(6.63 \mathrm{mmol})$ was dissolved in DMF and stirred under argon atmosphere for $10 \mathrm{~min}$ before sulfur trioxide pyridine complex $(19.88 \mathrm{mmol}, 3$ equiv) was added. The reaction was stirred for $2 \mathrm{~h}$ under argon atmosphere, followed by cooling to $0{ }^{\circ} \mathrm{C}$ and the slow addition of cold saturated sodium bicarbonate $\left(100 \mathrm{~mL}, 0{ }^{\circ} \mathrm{C}\right)$ under vigorously stirring the solution. Subsequently, tetrabutylammonium hydrogensulfate (9.94 mmol, 1.5 equiv) was added and the $\mathrm{pH}$ was lowered to $\mathrm{pH} 5 / 6$ by the slow addition of cold $0.1 \mathrm{M}$ citric acid $\left(150 \mathrm{~mL}, 0{ }^{\circ} \mathrm{C}\right)$. The solution was extracted three times with chloroform, and the organic layer was evaporated. The product was redissolved in water/acetonitrile $\mathrm{pH} 8$ (by addition of $1 \mathrm{M} \mathrm{NaOH}$ ) and lyophilized twice to obtain a white solid in $82.9 \%$ yield $(4.0 \mathrm{~g})$. LC-MS: $\mathrm{M} w_{\text {calc }}=482.48 \mathrm{~g} / \mathrm{mol}$ (Fmoc$\left.\mathrm{Y}\left(\mathrm{SO}_{3}\right)-\mathrm{OH}\right), \mathrm{M} w_{\text {calc }}=242.47 \mathrm{~g} / \mathrm{mol}\left(\mathrm{N}^{+} \mathrm{Bu}_{4}\right), \mathrm{m} / z_{\text {obs }}=242.42$ $\left[\mathrm{NBu}_{4}\right]^{+}, 482.58\left[\text { Fmoc- } \mathrm{Y}\left(\mathrm{SO}_{3}\right)-\mathrm{OH}\right]^{-}$.

Synthesis of Sulfated Serine for SP2. Fmoc-Ser $(\mathrm{tBu})-\mathrm{OH}(13.08$ $\mathrm{mmol}$ ) was deprotected in a cleavage cocktail containing TFA, TIS and water (95:2.5:2.5) for $4 \mathrm{~h}$ at room temperature. Subsequently, the TFA was evaporated and the residue was redissolved in water/ acetonitrile and lyophilized. A yellow oil was obtained in $116 \%$ yield $(4.96 \mathrm{~g})$ with traces of solvents. LC-MS: $\mathrm{M} w_{\text {calc }}=327.33 \mathrm{~g} / \mathrm{mol}, \mathrm{m} /$ $z_{\mathrm{obs}}=328.00[\mathrm{M}+\mathrm{H}]^{+}$. Subsequently, Fmoc-Ser $(\mathrm{OH})-\mathrm{OH}(9.17$ $\mathrm{mmol}$ ) was dissolved in DMF and stirred under argon atmosphere for $15 \mathrm{~min}$ before sulfur trioxide pyridine complex $(27.50 \mathrm{mmol}, 3$ equiv) was added. The reaction was stirred for $3 \mathrm{~h}$ under argon atmosphere, followed by cooling to $0{ }^{\circ} \mathrm{C}$ and the slow addition of cold saturated sodium bicarbonate $\left(60 \mathrm{~mL}, 0{ }^{\circ} \mathrm{C}\right)$ under vigorously stirring the solution. Subsequently, tetrabutylammonium hydrogensulfate (13.75 mmol, 1.5 equiv) was added and the $\mathrm{pH}$ was lowered to $\mathrm{pH} 5 / 6$ by the slow addition of cold $0.1 \mathrm{M}$ citric acid $\left(10 \mathrm{~mL}, 0{ }^{\circ} \mathrm{C}\right)$. The solution was extracted three times with chloroform, and the organic layer was evaporated. The product was redissolved in water/acetonitrile $\mathrm{pH} 8$ (by addition of $1 \mathrm{M} \mathrm{NaOH}$ ) and lyophilized twice to obtain an oil in $109 \%$ yield $(6.5 \mathrm{~g})$ with traces of solvent. $\mathrm{LC}-\mathrm{MS}: \mathrm{M} w_{\text {calc }}=406.39 \mathrm{~g} /$ $\mathrm{mol}\left(\mathrm{Fmoc}-\mathrm{S}\left(\mathrm{SO}_{3}\right)-\mathrm{OH}\right), \mathrm{M} w_{\text {calc }}=242.47 \mathrm{~g} / \mathrm{mol}\left(\mathrm{N}^{+} \mathrm{Bu}_{4}\right), m / z_{\text {obs }}=$ $242.33\left[\mathrm{NBu}_{4}\right]^{+}, 406.33\left[\mathrm{Fmoc}-\mathrm{Y}\left(\mathrm{SO}_{3}\right)-\mathrm{OH}\right]^{-}$.

Synthesis of Peptide Derivatives UF, SP1, SP2, and SP3. GSYDYG (UF), GSY $\left(\mathrm{OSO}_{3}^{-}\right) \mathrm{DY}\left(\mathrm{OSO}_{3}^{-}\right) \mathrm{G}(\mathrm{SP} 1), \mathrm{GSS}\left(\mathrm{OSO}_{3}^{-}\right) \mathrm{DS}\left(\mathrm{OSO}_{3}^{-}\right)$$\mathrm{G}(\mathrm{SP} 2)$, and $\mathrm{GSF}\left(\mathrm{SO}_{3} \mathrm{H}\right) \mathrm{DF}\left(\mathrm{SO}_{3} \mathrm{H}\right) \mathrm{G}(\mathrm{SP} 3)$ peptides were manually prepared using solid phase peptide synthesis (SPPS), Fmoc chemistry, and Rink amide MBHA resin. The $250 \mu \mathrm{mol}$ resin was allowed to swell in NMP for $1 \mathrm{~h}$. Subsequently, the resin was washed with NMP $(6 \times)$ and deprotected twice with a $20 \%$ piperidine solution in NMP for 5 min. The impurities were washed away with NMP $(6 \times)$ and a cocktail of the desired amino acid was prepared of $200 \mathrm{mM}$ amino acid, 1600 mM DIPEA, and 0.4 M HBTU in NMP (2:1:1, 5:2.5:2.5 mL). The cocktail was added to the resin and coupled for $30 \mathrm{~min}$ at room temperature. This cycle of washing-deprotection-washing-coupling was repeated for every amino acid. After the last amino acid was coupled, the remaining Fmoc protecting group was removed and the resin was washed extensively with NMP and DCM, followed by drying. Cleavage of the resin and amino acid protecting groups was performed in TFA/ $\mathrm{H}_{2} \mathrm{O} /$ TIS (94:2.5:2.5) for $2 \mathrm{~h}$ on ice (except for SP3 and UF, which was $4 \mathrm{~h}$ at room temperature). Subsequently, the peptides were precipitated in $20 \%$ cold hexane/diethyl ether, incubated for $15 \mathrm{~min}$ at $-20{ }^{\circ} \mathrm{C}$, and centrifuged for $10 \mathrm{~min}$ at $20 \mathrm{k}$ RPM. The supernatant was removed and the pellet was redissolved in water and lyophilized. The peptides (except UF) were purified using RP column chromatography using a gradient of $5-100 \%$ acetonitrile in water yielding the peptides in about $10 \%$ yield ( $98 \%$ yield for UF). SP1 and SP2 were defined as 

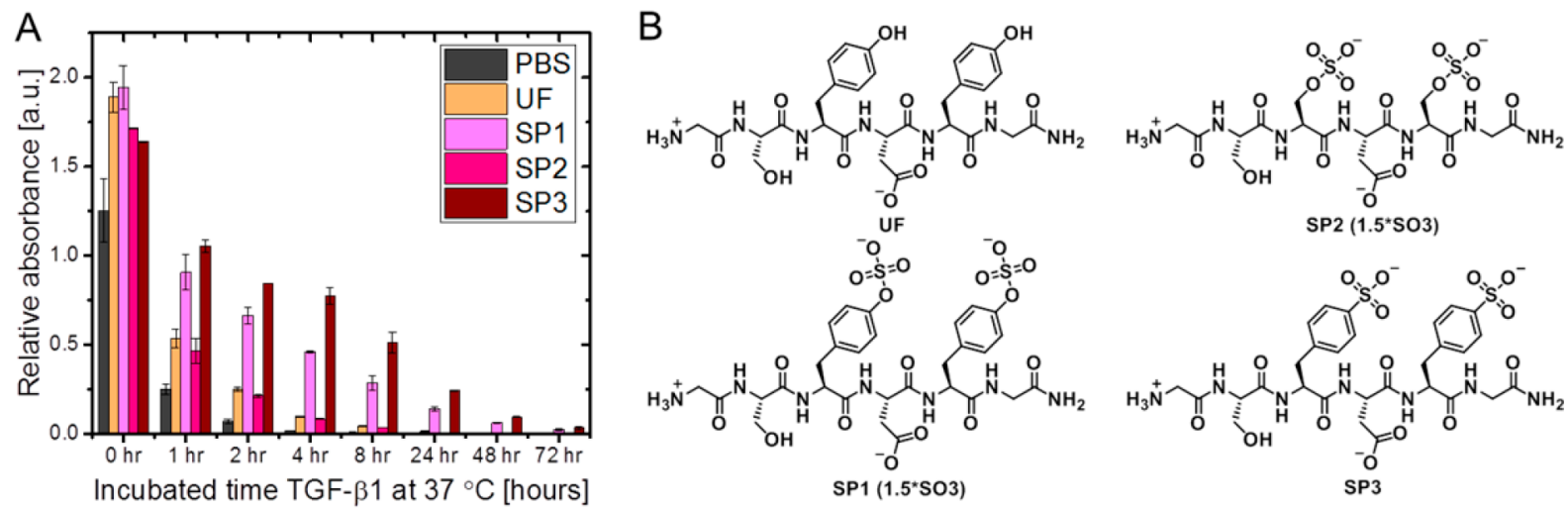

Figure 1. Peptide derivatives were assessed on their stabilization behavior on TGF- $\beta$ 1. (A) Optical density of TGF- $\beta 1$ after incubation with different peptide derivatives as measured with an ELISA assay. Sulfonated peptide SP3 showed the highest stabilizing effect $\left(c_{\text {TGF- } \beta 1} \approx 4 \mathrm{ng} / \mathrm{mL}, c_{\text {peptide }}=100\right.$ $\mu \mathrm{M}, \mathrm{c}_{\mathrm{BSA}} \approx 0.8 \mu \mathrm{g} / \mathrm{mL}, N=2$, error bars indicate standard deviation). (B) Molecular structures of the peptide derivatives assuming a $\mathrm{pH}$ of 7.4 .

having about 1.5 sulfates present based on LC-MS interpretation. LC-MS: $\mathrm{M} W_{\text {calc UF }}=659.65 \mathrm{~g} / \mathrm{mol}, \mathrm{m} / z_{\text {obs UF }}=660.42[\mathrm{M}+\mathrm{H}]^{+}$, $\mathrm{M} W_{\text {calc SP1 }}=817.65 \mathrm{~g} / \mathrm{mol}, \mathrm{m} / z_{\text {obs SP1 }}=818.25[\mathrm{M}-\mathrm{H}]^{-}, 408.75[\mathrm{M}-$ $2 \mathrm{H}]^{-}$, and $738.33\left[\mathrm{M}-\mathrm{SO}_{3}\right]^{-}, \mathrm{MW}_{\text {calc SP2 }}=665.56 \mathrm{~g} / \mathrm{mol}, \mathrm{m} / \mathrm{z}_{\text {obs SP2 }}=$

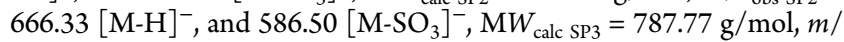
$z_{\text {obs SP } 3}=786.67[\mathrm{M}-\mathrm{H}]^{-}$, and $393.00[\mathrm{M}-2 \mathrm{H}]^{2-}$.

Synthesis of Supramolecular Peptide Conjugate UPy-SP3. The $\mathrm{UPy}-\mathrm{COOH}$ precursor molecule was synthesized as previously reported. ${ }^{25}$ The peptide resin $(31.0 \mu$ mole $)$ was allowed to swell for $1 \mathrm{~h}$ in DMF. Meanwhile, the reaction mixture containing UPy-COOH (60 mg, $52.7 \mu \mathrm{mol}, 1.7$ equiv), HATU ( $43.4 \mu \mathrm{mol}, 1.4$ equiv), DIPEA (93.0 $\mu \mathrm{mol}, 3$ equiv), and $4 \mathrm{~mL}$ of DMF was preactivated for $30 \mathrm{~min}$. Subsequently, the preactivated reaction mixture was added to the peptide resin and agitated overnight at room temperature. Afterward, the resin was washed with DMF $(6 \times)$ and DCM $(6 \times)$ and cleaved from the resin using a cocktail mixture containing TFA/TIS/H2O $(94: 2.5: 2.5)$ for $4 \mathrm{~h}$ at room temperature. The UPy-peptide was precipitated in cold $50 \%$ hexane/ether, incubated for $15 \mathrm{~min}$ at -20 ${ }^{\circ} \mathrm{C}$ and centrifuged for $10 \mathrm{~min}$ at $20 \mathrm{k}$ RPM. The supernatant was removed, the pellet was redissolved in $10 \%$ acetonitrile/water and the solvent was lyophilized. Purification with RP column chromatography using a gradient of $5-100 \%$ acetonitrile in water yielded in pure UPySP3 (10.1 mg, $17.1 \%$ yield). LC-MS: $M W_{\text {calc }}=1908.14 \mathrm{~g} / \mathrm{mol}, \mathrm{m} / z_{\text {obs }}$ $=954.4[\mathrm{M}-2 \mathrm{H}]^{2-}$.

ELISA Experiments. Samples were dissolved in PBS in the right concentration. Because of the undefined state of both SP1 and SP2, a molecular weight of $738.70\left(1 \mathrm{OSO}_{3}^{-}\right)$and $626.03 \mathrm{~g} / \mathrm{mol}\left(1.5 \mathrm{OSO}_{3}{ }^{-}\right)$ was used, respectively. The UPy samples were prepared by heating the solid dissolved in PBS to $72{ }^{\circ} \mathrm{C}$ for $1 \mathrm{~h}$, and UPy-SP3 was incorporated by an additional incubation step of $15 \mathrm{~min}$ at $45{ }^{\circ} \mathrm{C}$. Annealing overnight at room temperature resulted in self-assembled polymers with UV patterns similar to reference spectra. ${ }^{26}$ A stock of every condition was prepared by the addition of TGF- $\beta 1(0.8 \mu \mathrm{L}$ for $1 \mathrm{~mL}$ to obtain a $4 \mathrm{ng} / \mathrm{mL}$ stock, or $0.5 \mu \mathrm{L}$ for $1 \mathrm{~mL}$ to obtain a $2.5 \mathrm{ng} / \mathrm{mL}$ stock). For each condition, $100 \mu \mathrm{L}$ was pipetted into a 96 -well plate (in duplicate or triplicate for each time point), incubated at $37^{\circ} \mathrm{C}$ with $5 \% \mathrm{CO}_{2}$, removed at indicated time points, and stored at $-20^{\circ} \mathrm{C}$ until the ELISA experiment was conducted. The ELISA experiments were performed following the manufacturer's protocol. Briefly, standard or sample was incubated for $2 \mathrm{~h}$, washed four times with wash buffer, incubated with growth factor specific conjugate for $2 \mathrm{~h}$, washed four times with wash buffer, and incubated for $30 \mathrm{~min}$ with substrate solution. Then the reaction was quenched with stop solution. The optical density was measured at $450 \mathrm{~nm}$ with a wavelength correction set to $570 \mathrm{~nm}$.

Cell Culture. Human fibrosarcoma cells (HT1080, kindly provided by Marie-José Goumans), transfected with (CAGA) ${ }_{9}$ MLP-luc using FuGENE6, ${ }^{27}$ were cultured in DMEM (Gibco) supplemented with $1 \%$ penicillin/streptomycin (Lonza), 10\% fetal bovine serum (Bovogen), and $1 \%$ nonessential amino acids. Cells were routinely cultured at 37
${ }^{\circ} \mathrm{C}$ and $5 \% \mathrm{CO}_{2}$. Medium was changed every $2-3$ days and passaged at $80-90 \%$ confluency. This cell line expresses luciferase upon exposure to extracellular TGF- $\beta 1$, which is regulated via Smad3/ Smad4 signaling and binding to the CAGA box located in the plasminogen activator inhibitor-1 (PAI-1) gene.

Luciferase Assay. HT1080 cells were seeded at a density of 50000 cell $/ \mathrm{cm}^{2}$ in a 12 -well plate and allowed to adhere overnight at $37^{\circ} \mathrm{C}$ and $5 \% \mathrm{CO}_{2}$. The excipient molecules were dissolved in DMEM (containing $1 \%$ pen/strep) in the correct concentration. The UPy samples were prepared by heating the solution to $72{ }^{\circ} \mathrm{C}$ for $1 \mathrm{~h}$, and UPy-SP3 was incorporated by an additional incubation step of $15 \mathrm{~min}$ at $45{ }^{\circ} \mathrm{C}$. Annealing overnight at room temperature resulted in selfassembled polymers with similar UV patterns. The next day, medium was replaced by serum-free DMEM supplemented with $1 \%$ pen/strep and incubated for $7 \mathrm{~h}$. A stock of every condition was prepared by the addition of TGF- $\beta 1$ ( $0.8 \mu \mathrm{L}$ for $1 \mathrm{~mL}$ to obtain a $4 \mathrm{ng} / \mathrm{mL}$ stock, or $0.5 \mu \mathrm{L}$ for $1 \mathrm{~mL}$ to obtain a $2.5 \mathrm{ng} / \mathrm{mL}$ stock). Four hours before cell seeding, $1.2 \mathrm{~mL}$ of the stock solutions was pipetted (in triplicate) in a plain 12 wells plate and incubated at $37{ }^{\circ} \mathrm{C}$ with $5 \% \mathrm{CO}_{2}$ to yield into the $4 \mathrm{~h}$ preincubated samples. Subsequently, either $1 \mathrm{~mL}$ fresh stock was added onto the cells or $1 \mathrm{~mL}$ of the $4 \mathrm{~h}$ preincubated samples (in triplicate). Cells were then incubated an additional $19 \mathrm{~h}$, lysed, and the luciferase expression was measured (in duplicate of the triplicate) according to the manufacturer's protocol (Promega). Relative luciferase intensities were normalized for the DNA content using the CyQUANT cell proliferation assay (Invitrogen).

\section{RESULTS AND DISCUSSION}

Selection of Peptide Derivative. The stability of the growth factor TGF- $\beta 1$ was studied over time, and heparin peptide mimics were selected to assess their binding. TGF- $\beta 1$ is known to modulate behavior of many cell types including immune cells. Furthermore, altered signaling is associated with several disorders such as cancer and fibrosis. ${ }^{28,29}$ TGF- $\beta 1$ is synthesized as an inactive precursor, which is activated upon cleavage from the ECM by proteases followed by acidic conditions to remove the latent TGF- $\beta$ binding protein. ${ }^{30} \mathrm{We}$ assessed the stability of activated TGF- $\beta 1$ over time at $37{ }^{\circ} \mathrm{C}$ with an enzyme-linked immunosorbent assay (ELISA) revealing full degradation within $5 \mathrm{~h}$ (Figure 1A, PBS condition, half-life of about $0.4 \mathrm{~h}$ ). Then four peptide derivatives, based on the tetrapeptide of Maynard and Hubbell (here named SP1), ${ }^{20}$ were synthesized and assessed for their ability to stabilize TGF$\beta 1$ (Figure 1). In this small library, unfunctionalized peptide (UF) was included to investigate the absence of the sulfate groups. Sulfated peptide 2 (SP2) lacks aromatic rings hence hydrophobic effects as compared to sulfated peptide 1 (SP1), 


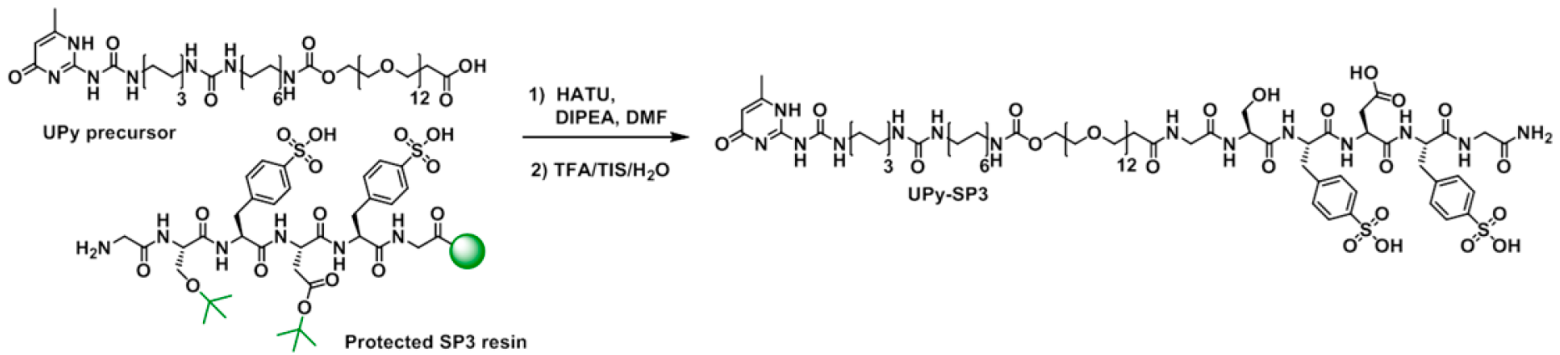

Figure 2. Synthesis of the UPy-SP3 conjugate. The UPy precursor was coupled onto the peptide resin, followed by the cleavage of the protecting groups and the resin (green).
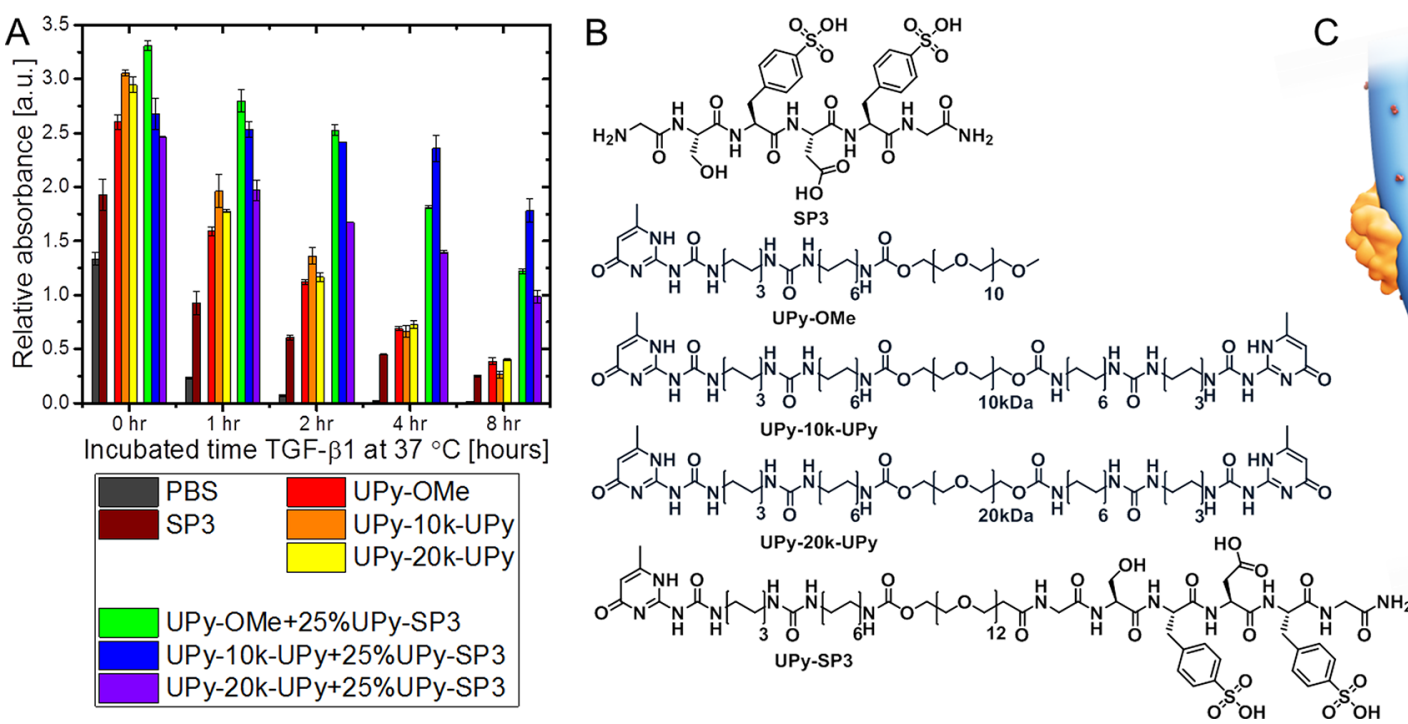

Figure 3. Effect of supramolecular polymers on TGF- $\beta 1$ stabilization. (A) Optical density of TGF- $\beta 1$ after incubation with different supramolecular excipients as measured with an ELISA assay. Physical entanglement of TGF- $\beta 1$ with bare UPy fibers showed a similar stabilizing effect as compared to SP3, whereas the incorporation of UPy-SP3 in supramolecular fibers enhanced the stabilization significantly $\left(c_{\mathrm{TGF}-\beta 1} \approx 4 \mathrm{ng} / \mathrm{mL}, c_{\text {peptide }}=100 \mu \mathrm{M}\right.$, $c_{\mathrm{UPy}}=400 \mu \mathrm{M}, c_{25 \% \mathrm{UPy}-\mathrm{SP} 3}$ in UPy $=100 \mu \mathrm{M}, c_{\mathrm{BSA}} \approx 0.8 \mu \mathrm{g} / \mathrm{mL}, N=2$, error bars indicate standard deviation). (B) Molecular structures of the excipient molecules. (C) Cartoon representing the proposed binding of TGF- $\beta 1$ (orange) to supramolecular polymers (blue) coassembled with sulfonated peptides (red).

and finally, since sulfate groups are labile, a more stable sulfonated peptide (SP3) was included.

The four peptides were synthesized with manual solid phase peptide synthesis using sulfated or sulfonated amino acids. Because of the acid sensitive sulfate groups, ${ }^{31}$ both SP1 and SP2 were difficult to obtain in a fully defined state (about 1.5 sulfate groups instead of 2 were obtained). The purified excipient peptides were mixed with TGF- $\beta 1$ and incubated for different time points (Figure $1 \mathrm{~A}$ and Supporting Information, Figure S1). In the ELISA, UF and SP2 showed a low relative absorbance whereas SP1 and SP3 showed a more enhanced optical density over time. The lower stabilization effect of SP1 might be due to the labile sulfate group or the less-defined state of only 1-1.5 sulfate groups per molecule instead of the maximum of 2 , and the presence of more salt as counterions yielding a slightly lower concentration as intended. Peptide SP2 and the UF peptide showed similar behavior highlighting the importance of both a sulfate or sulfonate and an aromatic ring for TGF- $\beta 1$ binding. All peptides showed a significant enhancement of the half-life of TGF- $\beta 1$, of which SP3 was shown to have the most pronounced stabilization potential of the peptides studied. Moreover, increasing or decreasing the concentration of the excipient peptides has an enhanced or reduced effect, respectively (Supporting Information, Figure
S2). In addition, it was shown that SP1 at $10 \mu \mathrm{M}$ has a higher stabilizing effect as compared to heparin. This introductory study indicated that a combination of hydrophobic effects, hydrogen bonding, and electrostatic effects contribute to growth factor binding and is concentration dependent.

Implementation into the Supramolecular Platform. Subsequently, the sulfonated peptide SP3 was integrated in a UPy-based supramolecular system to investigate increased local concentration on the stabilization of TGF- $\beta 1$. It was previously shown that a subtle change in the design and coassembly of the supramolecular polymer system has a profound effect on the internal dynamics of the system. ${ }^{26}$ Moreover, the size of the hydration shell of the ethylene glycol might have an effect on growth factor stabilization as well. Therefore, three different UPy scaffolding molecules (UPy-OMe, UPy-10k-UPy, and UPy-20k-UPy, Figure 3B) were used. SP3 was coupled onto a UPy precursor molecule to allow incorporation in the scaffolding molecules by coassembly (Figure 2). Different percentages of UPy-SP3 conjugate were coassembled into monovalent UPy stacks and the stacking behavior was investigated using UV-vis spectroscopy (Supporting Information, Figure S3). Since the absorbance of the peptide overlapped with the absorption spectrum of the UPy, bare SP3 at different concentrations was measured and subtracted 

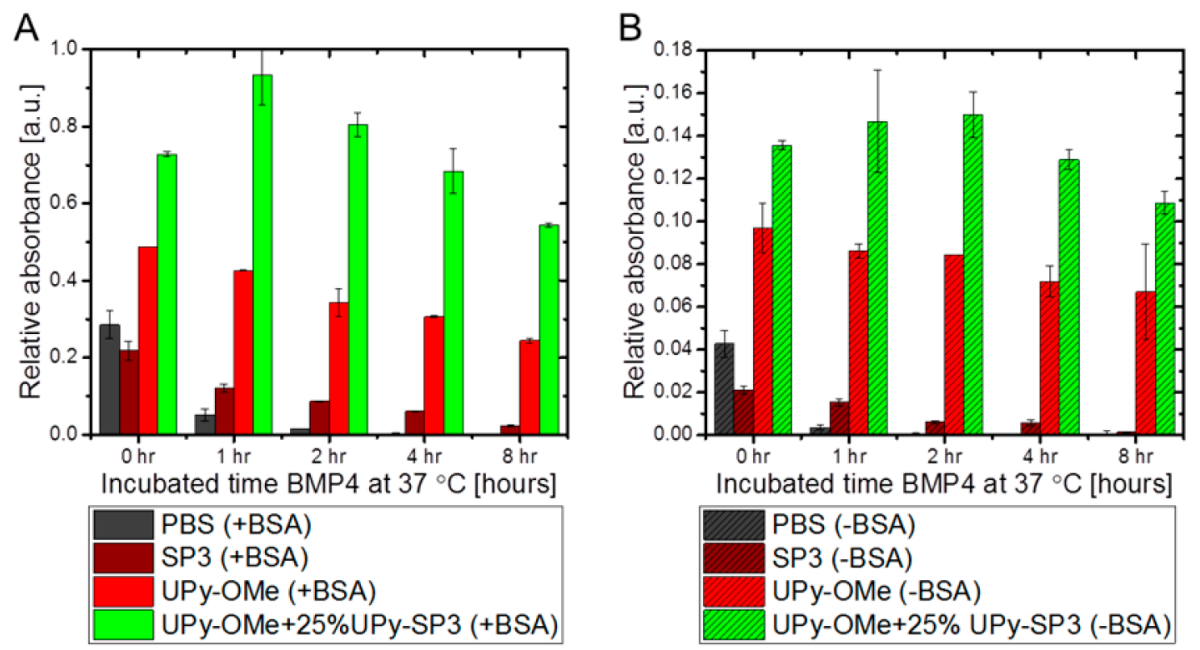

Figure 4. Supramolecular polymers also stabilize BMP4 (A) with BSA (+BSA) and (B) without BSA (-BSA). Without BSA, BMP4 has a low stability; however, when mixed with supramolecular polymers and BSA, the activity of BMP4 was enhanced for several hours $\left(c_{\mathrm{BMP} 4} \approx 2.5 \mathrm{ng} / \mathrm{mL}\right.$, $c_{\text {peptide }}=62.5 \mu \mathrm{M}, c_{\mathrm{UPy}}=250 \mu \mathrm{M}, c_{\mathrm{BSA}} \approx 0.5 \mu \mathrm{g} / \mathrm{mL}$, error bars indicate standard deviation, $N=2$ ).

from the corresponding coassembled spectra. The spectra obtained after subtraction overlapped with each other, thus indicating the successful incorporation, though increased intensity at $270 \mathrm{~nm}$ for $100 \%$ UPy-SP3 was observed. This is probably due to steric and repulsive effects of the peptides generating smaller and less defined aggregates at a $100 \%$ functionalization. As a result, a coassembly percentage of 25 mol \% was chosen to include in a TGF- $\beta 1$ stabilization assay. The three bare UPy scaffolding molecules (i.e., without UPySP3) were also included to investigate the effect of physical adsorption on TGF- $\beta 1$ stabilization.

Investigation of TGF- $\beta 1$ Stabilization. TGF- $\beta 1$ was mixed with preassembled UPy samples with different compositions and incubated for different time points (Figure 3, and Supporting Information, Figure S4). The concentration of the functional epitope was kept constant $\left(c_{\mathrm{SP} 3}=c_{\mathrm{UPy}-\mathrm{SP} 3}=\right.$ $100 \mu \mathrm{M})$ resulting in a total UPy concentration of $400 \mu \mathrm{M}$. All studies were performed in solution; however, the UPy-10k-UPy excipient formed a thin viscous layer on the bottom of the well plate indicative of minor gel formation at a $400 \mu \mathrm{M}$ concentration. For the bare UPy scaffolds (UPy-OMe, UPy10k-UPy, and UPy-20k-UPy), a slightly higher relative absorbance over time was observed as compared to the reference SP3, probably due to the four-times higher concentration. Upon incorporating $25 \mathrm{~mol} \%$ of UPy-SP3 in the different scaffolding monomers, a synergistic effect was observed, with the highest stabilizing effect of UPy-10k-UPy scaffold with $25 \mathrm{~mol} \%$ UPy-SP3 incorporated. The same trend was observed at lower concentration TGF- $\beta 1(2.5 \mathrm{ng} / \mathrm{mL})$ mixed with 1.6 fold lower UPy concentration (Supporting Information, Figure S5).

The binding of TGF- $\beta 1$ to the excipient molecules was proposed to occur via electrostatic interactions to binding sites at the interface between the TGF- $\beta 1$ dimers rather than wrapping around the growth factor based on previous studies $^{32,33}$ (Figure 3C). In contrast, binding to nonfunctionalized supramolecular polymers might be due to nonspecific PEG binding or hydrophobic interactions. Importantly, growth factor binding has a minor influence on the stacking behavior as shown with UV-vis spectroscopy (Supporting Information, Figure S6). Moreover, it was proposed that due to the temporal noncovalent interactions between the excipient and growth factor, dissociation of the growth factor might result in presentation to the cell receptor (important for in vitro assays) or prone to conformational changes due to its free occurrence in solution or adherence to the plastic environment leading to denaturation. Nevertheless, the results obtained here indicate that the half-life of TGF- $\beta 1$ was significantly enhanced by binding to the UPy fibers without changing the fiber organization.

Investigation of BMP4 Stabilization. To investigate whether the excipient molecules are also applicable to other growth factors, the stabilization of bone morphogenic protein- 4 (BMP4) was evaluated (Figure 4, and Supporting Information, Figures S7 and S8). BMP4 plays an important role in cardiac development during the embryonic stage and it is known to be upregulated during impaired remodeling of the adult heart. ${ }^{34,35}$ Despite being a member of the transforming growth factor beta superfamily with similarities in secondary structure, binding of BMP4 to heparan sulfate might occur on different positions compared to TGF- $\beta 1 .^{36}$ Therefore, excipient binding and stabilization of BMP4 might be altered. In addition to investigation of the stabilization of BMP4, also the influence of BSA was evaluated, since supraphysiological amounts of BSA are typically added to enhance growth factor stability. All previous experiments were carried out in the presence of BSA. Here, we investigate the stability of a growth factor both in the presence and in the absence of BSA. In both cases, with or without BSA added, growth factor binding by monovalent UPyOMe functionalized with $25 \mathrm{~mol} \%$ UPy-SP3 showed a synergistic effect as compared to the nonfunctionalized supramolecular polymer (UPy-OMe) and reference SP3, which remained relatively constant over a few hours (Figure $4 \mathrm{~A}$ and $\mathrm{B}$, respectively). Interestingly, when BSA was added, the relative absorbance of BMP4 after $1 \mathrm{~h}$ was slightly higher than the $0 \mathrm{~h}$ sample, probably due to experimental error or conformational changes of the UPy at $37{ }^{\circ} \mathrm{C}$ leading to more optimal binding hence stabilization. Without BSA, BMP4 has a low stability, with about six-fold lower initial relative absorbance as compared to BMP4 containing BSA $\left(c_{\mathrm{BSA}} \approx 0.5 \mu \mathrm{g} / \mathrm{mL}\right.$, Figure $4 \mathrm{~B})$. This lower concentration could be explained by the acid treatment necessary to activate BMP4, which most likely degraded BMP4 much more in the absence of BSA. As a result, BSA has a strong and short initial stabilization effect which 

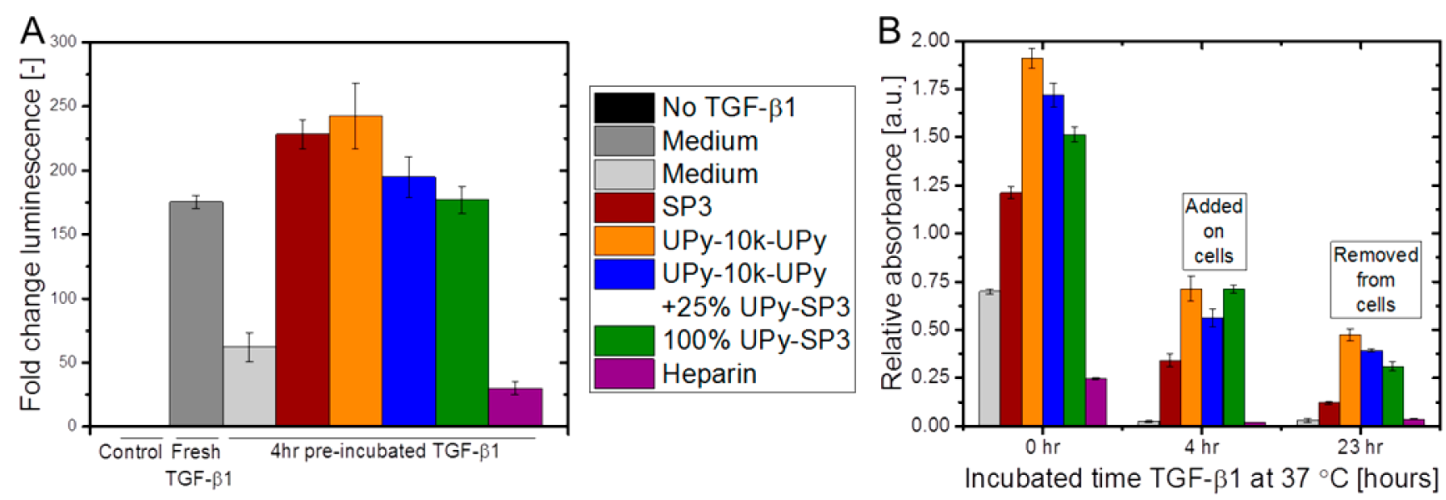

Figure 5. (A) TGF- $\beta 1$ response after cell incubation in the form of luciferase and (B) corresponding optical density of TGF- $\beta 1$ as measured with ELISA. In contrast to the cell experiments, the UPy samples have a slightly higher TGF- $\beta 1$ optical density as compared to SP3 indicating that the PEG shell decreases TGF- $\beta 1$ receptor presentation $\left(c_{\text {TGF }-\beta 1} \approx 2.5 \mathrm{ng} / \mathrm{mL}, c_{\text {peptide }}=62.5 \mu \mathrm{M}, c_{\text {UPy }}=250 \mu \mathrm{M}, c_{\mathrm{BSA}} \approx 0.5 \mu \mathrm{g} / \mathrm{mL}\right.$, error bars indicate standard deviation, (A) $N=3$, (B) $N=2$, (A) data was corrected for the DNA content).

quickly diminishes after an hour. In contrast, upon addition of the supramolecular platform after activation, the percentage active BMP4 was better retained. Therefore, the stability of BMP4 might be further enhanced when the supramolecular platform would be added during growth factor activation, that is, acid treatment.

Cellular Readout of Active TGF- $\beta 1$. To investigate, as a proof-of-principle, whether the stabilized TGF- $\beta 1$ with excipient molecules is still able to induce cellular responses, HT1080 fibrosarcoma cells were exposed to different conditions. These cells have a luciferase reporter, which can convert active TGF- $\beta 1$ into luciferase. ${ }^{27}$ The converted amount of luciferase is directly proportional to the amount of active TGF- $\beta 1$. Moreover, the luciferase activity corresponds to the initial concentration of TGF- $\beta 1$ rather than the concentration during incubation with the cells (Supporting Information, Figures S9 and S10). For this reason, excipient molecules mixed with TGF- $\beta 1$ were preincubated for $4 \mathrm{~h}$ prior to cell exposure (2.5 ng/mL TGF- $\beta 1,62.5 \mu \mathrm{M} \mathrm{SP3}$, and $250 \mu \mathrm{M}$ UPy, Figure 5, and Supporting Information, Figures S11 and S12). As expected, a high luciferase activity was observed when the cells were exposed to fresh TGF- $\beta 1$ (i.e., TGF- $\beta 1$ addition directly after thawing) and the activity decreased when TGF- $\beta 1$ was preincubated before addition to cells (Figure 5A). Both SP3 and UPy-10k-UPy showed the highest luciferase activity. However, ELISA clearly confirmed the presence of more active TGF- $\beta 1$ when mixed with UPy excipients (UPy-10k-UPy + 25\% UPy-SP3 and 100\% UPy-SP3, Figure 5B). This might be due to the poly(ethylene glycol) surrounding the fibers, which can partly shield the TGF- $\beta 1$ presentation toward cells owing to their hydration shell. ${ }^{37}$ Although the $100 \%$ UPy-SP3 has the same concentration as the reference experiment with SP3, which should increase the local concentration, a lower cellular response was observed in contradiction to the ELISA results. Because of multivalent effects, the binding affinity to the excipient might be increased, lowering receptor binding availability. Moreover, a thin layer of gel was observed on the bottom of the well plate (during preincubation) for the UPy samples, which might contain local higher growth factor concentration. Though this layer was not transferred onto the cells, probably some TGF- $\beta 1$ was lost during this step. Interestingly, heparin even showed a lower cellular response than PBS, in corroboration with the ELISA results. The reason that heparin has no stabilizing effect might be due to a suboptimal sulfate-pattern present, failing to support TGF- $\beta 1$ binding. This observation is supported by a study of Gallagher et al. where heparan sulfate originating from porcine mucosa was proved to have low binding affinity. ${ }^{33}$

Taking the results all together, a strong cellular response was observed in all cases. However, coassembling the sulfonated peptide in the supramolecular platform (UPy-10k-UPy $+25 \%$ UPy-SP3) did not increase the luciferase activity as compared to the bare supramolecular polymer (UPy-10k-UPy) when TGF- $\beta 1$ was preincubated for $4 \mathrm{~h}$, though more active TGF- $\beta 1$ was detected with ELISA. Probably the concentration plays an important role, which is in this case close to receptor saturation levels (Supporting Information, Figure S9), and also the preincubation time could be extended to induce more differences between the conditions. Further extensive studies are necessary to find the optimal concentration and incubation time for these cell experiments.

\section{CONCLUSION}

Materials based on supramolecular polymers are proposed to be ideal platforms for mimicking the natural extracellular matrix due to their dynamic, responsive and adaptable properties. Supramolecular polymers based on peptide amphiphiles functionalized with bioactive cues were already proven to be successful in binding growth factors. Here, we introduced a sulfonated peptide coassembled in ureidopyrimidinone-based supramolecular polymer platforms for the stabilization of the growth factor TGF- $\beta 1$. Different excipient molecules were shown to prolong the half-life of the growth factor, and proofof-principle cell experiments confirmed high cellular response in the form of luciferase, indicating the maintenance of TGF- $\beta 1$ activity upon excipient binding. Although there is a slight difference in cellular read-out and the corresponding ELISA experiments, maybe due to steric effects of the PEG preventing optimal cell presentation, all results show a significant stabilization in all experimental systems. Moreover, it was shown that the excipient molecules can also be used for the stabilization of other heparan sulfate binding proteins, as was revealed with BMP4. This approach can be used to extend the activity of important growth factors but can also be implemented in hydrogel systems. In hydrogel systems, the sustained release can be controlled by the binding strength to the protein and by tuning the physical properties of the hydrogel. Gaining more control over growth factor concentrations is beneficial in biomedical applications, for example, in 
directing specific processes during different stages of tissue regeneration.

\section{ASSOCIATED CONTENT}

\section{S Supporting Information}

The Supporting Information is available free of charge on the ACS Publications website at DOI: 10.1021/acs.biomac.8b00219.

LC-MS spectra of molecules, additional ELISA results, UV-vis experiments, statistical analysis (PDF)

\section{AUTHOR INFORMATION}

\section{Corresponding Authors}

*E-mail: E.W.meijer@tue.nl.

*E-mail: P.Y.W.Dankers@tue.nl.

ORCID $\odot$

E. W. Meijer: 0000-0003-4126-7492

Patricia Y. W. Dankers: 0000-0002-8997-181X

\section{Author Contributions}

The study was designed and the results were obtained by S.I.S.H. and S.S. The experiments were proposed and results were discussed by all authors. The manuscript was written through contributions of all authors. All authors have given approval to the final version of the manuscript.

\section{Notes}

The authors declare no competing financial interest.

\section{ACKNOWLEDGMENTS}

This work was funded by the NWO/DPI program NEWPOL (Project No. 731.015.503), partially financed with TKI (Topconsortia for Knowledge Innovation) allowance provided by the Dutch Ministry of Economic Affairs. E.W.M. acknowledges the Ministry of Education, Culture and Science (Gravity program FMS, 024.001.035). P.Y.W.D. acknowledges the European Research Council (FP7/2007-2013) ERC Grant Agreement 308045. We also acknowledge the support from The Netherlands Cardiovascular Research Initiative (CVON 2012-01), the Dutch Heart Foundation, Dutch Federation of University Medical Centers, The Netherlands Organization for Health Research and Development, and the Royal Netherlands Academy of Sciences. The authors thank Prof. Marie-José Goumans (Leiden University Medical Center, The Netherlands) for providing the HT1080 cells.

\section{REFERENCES}

(1) Rodrigues, M.; Griffith, L. G.; Wells, A. Growth factor regulation of proliferation and survival of multipotential stromal cells. Stem Cell Res. Ther. 2010, 1 (4), 32.

(2) Cipitria, A.; Salmeron-Sanchez, M. Mechanotransduction and Growth Factor Signalling to Engineer Cellular Microenvironments. Adv. Healthcare Mater. 2017, 6 (15), 1700052.

(3) Farach-Carson, M. C.; Carson, D. D. Perlecan-a multifunctional extracellular proteoglycan scaffold. Glycobiology 2007, 17 (9), 897905.

(4) Capila, I.; Linhardt, R. J. Heparin-Protein Interactions. Angew. Chem., Int. Ed. 2002, 41 (3), 390-412.

(5) Xu, D.; Esko, J. D. Demystifying Heparan Sulfate-Protein Interactions. Annu. Rev. Biochem. 2014, 83 (1), 129-157.

(6) Lee, K.; Silva, E. A.; Mooney, D. J. Growth factor delivery-based tissue engineering: general approaches and a review of recent developments. J. R. Soc., Interface 2011, 8 (55), 153-170.

(7) Francis, G. L. Albumin and mammalian cell culture: implications for biotechnology applications. Cytotechnology 2010, 62 (1), 1-16.
(8) Bian, L.; Zhai, D. Y.; Tous, E.; Rai, R.; Mauck, R. L.; Burdick, J. A. Enhanced MSC chondrogenesis following delivery of TGF- $\beta 3$ from alginate microspheres within hyaluronic acid hydrogels in vitro and in vivo. Biomaterials 2011, 32 (27), 6425-6434.

(9) Watarai, A.; Schirmer, L.; Thönes, S.; Freudenberg, U.; Werner, C.; Simon, J. C.; Anderegg, U. TGF $\beta$ functionalized starPEG-heparin hydrogels modulate human dermal fibroblast growth and differentiation. Acta Biomater. 2015, 25, 65-75.

(10) Paluck, S. J.; Nguyen, T. H.; Lee, J. P.; Maynard, H. D. A Heparin-Mimicking Block Copolymer Both Stabilizes and Increases the Activity of Fibroblast Growth Factor 2 (FGF2). Biomacromolecules 2016, 17 (10), 3386-3395.

(11) Mitchell, A. C.; Briquez, P. S.; Hubbell, J. A.; Cochran, J. R. Engineering growth factors for regenerative medicine applications. Acta Biomater. 2016, 30, 1-12.

(12) Freudenberg, U.; Liang, Y.; Kiick, K. L.; Werner, C. Glycosaminoglycan-Based Biohybrid Hydrogels: A Sweet and Smart Choice for Multifunctional Biomaterials. Adv. Mater. 2016, 28 (40), 8861-8891.

(13) Paluck, S. J.; Nguyen, T. H.; Maynard, H. D. HeparinMimicking Polymers: Synthesis and Biological Applications. Biomacromolecules 2016, 17 (11), 3417-3440.

(14) Lee, S. S.; Fyrner, T.; Chen, F.; Álvarez, Z.; Sleep, E.; Chun, D. S.; Weiner, J. A.; Cook, R. W.; Freshman, R. D.; Schallmo, M. S.; Katchko, K. M.; Schneider, A. D.; Smith, J. T.; Yun, C.; Singh, G.; Hashmi, S. Z.; McClendon, M. T.; Yu, Z.; Stock, S. R.; Hsu, W. K.; Hsu, E. L.; Stupp, S. I. Sulfated glycopeptide nanostructures for multipotent protein activation. Nat. Nanotechnol. 2017, 12 (8), 821829.

(15) Kocabey, S.; Ceylan, H.; Tekinay, A. B.; Guler, M. O. Glycosaminoglycan mimetic peptide nanofibers promote mineralization by osteogenic cells. Acta Biomater. 2013, 9 (11), 9075-9085.

(16) Mammadov, R.; Mammadov, B.; Guler, M. O.; Tekinay, A. B. Growth Factor Binding on Heparin Mimetic Peptide Nanofibers. Biomacromolecules 2012, 13 (10), 3311-3319.

(17) Yasa, O.; Uysal, O.; Ekiz, M. S.; Guler, M. O.; Tekinay, A. B. Presentation of functional groups on self-assembled supramolecular peptide nanofibers mimicking glycosaminoglycans for directed mesenchymal stem cell differentiation. J. Mater. Chem. B 2017, 5 (25), 4890-4900.

(18) Sidhu, P. S.; Mosier, P. D.; Zhou, Q.; Desai, U. R. On scaffold hopping: Challenges in the discovery of sulfated small molecules as mimetics of glycosaminoglycans. Bioorg. Med. Chem. Lett. 2013, 23 (1), $355-359$.

(19) Azevedo, H. S.; Pashkuleva, I. Biomimetic supramolecular designs for the controlled release of growth factors in bone regeneration. Adv. Drug Delivery Rev. 2015, 94, 63-76.

(20) Goor, O. J. G. M.; Hendrikse, S. I. S.; Dankers, P. Y. W.; Meijer, E. W. From supramolecular polymers to multi-component biomaterials. Chem. Soc. Rev. 2017, 46 (21), 6621-6637.

(21) Shah, R. N.; Shah, N. A.; Del Rosario Lim, M. M.; Hsieh, C.; Nuber, G.; Stupp, S. I. Supramolecular design of self-assembling nanofibers for cartilage regeneration. Proc. Natl. Acad. Sci. U. S. A. 2010, 107 (8), 3293-3298.

(22) Maynard, H. D.; Hubbell, J. A. Discovery of a sulfated tetrapeptide that binds to vascular endothelial growth factor. Acta Biomater. 2005, 1 (4), 451-459.

(23) Kim, S. H.; Kiick, K. L. Heparin-mimetic sulfated peptides with modulated affinities for heparin-binding peptides and growth factors. Peptides 2007, 28 (11), 2125-2136.

(24) Dankers, P. Y. W.; Hermans, T. M.; Baughman, T. W.; Kamikawa, Y.; Kieltyka, R. E.; Bastings, M. M. C.; Janssen, H. M.; Sommerdijk, N. A. J. M.; Larsen, A.; van Luyn, M. J. A.; Bosman, A. W.; Popa, E. R.; Fytas, G.; Meijer, E. W. Hierarchical Formation of Supramolecular Transient Networks in Water: A Modular Injectable Delivery System. Adv. Mater. 2012, 24 (20), 2703-2709.

(25) de Feijter, I.; Goor, O. J. G. M.; Hendrikse, S. I. S.; ComellasAragonès, M.; Söntjens, S. H. M.; Zaccaria, S.; Fransen, P. P. K. H.; Peeters, J. W.; Milroy, L.-G.; Dankers, P. Y. W. Solid-Phase-Based 
Synthesis of Ureidopyrimidinone-Peptide Conjugates for Supramolecular Biomaterials. Synlett 2015, 26 (19), 2707-2713.

(26) Hendrikse, S. I. S.; Wijnands, S. P. W.; Lafleur, R. P. M.; Pouderoijen, M. J.; Janssen, H. M.; Dankers, P. Y. W.; Meijer, E. W. Controlling and tuning the dynamic nature of supramolecular polymers in aqueous solutions. Chem. Commun. 2017, 53 (14), 2279-2282.

(27) Dennler, S.; Itoh, S.; Vivien, D.; ten Dijke, P.; Huet, S.; Gauthier, J. M. Direct binding of Smad3 and Smad4 to critical TGF $\beta$ inducible elements in the promoter of human plasminogen activator inhibitor-type 1 gene. EMBO J. 1998, 17 (11), 3091-3100.

(28) Weiss, A.; Attisano, L. The TGFbeta Superfamily Signaling Pathway. Wiley Interdiscip. Rev.: Dev. Biol. 2013, 2 (1), 47-63.

(29) Dennler, S.; Goumans, M.-J.; ten Dijke, P. Transforming growth factor $\beta$ signal transduction. J. Leukocyte Biol. 2002, 71 (5), 731-740.

(30) Kubiczkova, L.; Sedlarikova, L.; Hajek, R.; Sevcikova, S. TGF- $\beta$ - an excellent servant but a bad master. J. Transl. Med. 2012, 10 (1), 183.

(31) Balsved, D.; Bundgaard, J. R.; Sen, J. W. Stability of tyrosine sulfate in acidic solutions. Anal. Biochem. 2007, 363 (1), 70-76.

(32) Lee, J.; Wee, S.; Gunaratne, J.; Chua, R. J. E.; Smith, R. A. A.; Ling, L.; Fernig, D. G.; Swaminathan, K.; Nurcombe, V.; Cool, S. M. Structural determinants of heparin-transforming growth factor- $\beta 1$ interactions and their effects on signaling. Glycobiology 2015, 25 (12), 1491-1504.

(33) Lyon, M.; Rushton, G.; Gallagher, J. T. The Interaction of the Transforming Growth Factor- $\beta$ s with Heparin/Heparan Sulfate Is Isoform-specific. J. Biol. Chem. 1997, 272 (29), 18000-18006.

(34) Talkhabi, M.; Aghdami, N.; Baharvand, H. Human cardiomyocyte generation from pluripotent stem cells: A state-of-art. Life Sci. 2016, 145, 98-113.

(35) Guo, W.-T.; Dong, D.-L. Bone morphogenetic protein-4: a novel therapeutic target for pathological cardiac hypertrophy/heart failure. Heart Failure Rev. 2014, 19 (6), 781-788.

(36) Gandhi, N. S.; Mancera, R. L. Prediction of heparin binding sites in bone morphogenetic proteins (BMPs). Biochim. Biophys. Acta, Proteins Proteomics 2012, 1824 (12), 1374-1381.

(37) Chen, S.; Li, L.; Zhao, C.; Zheng, J. Surface hydration: Principles and applications toward low-fouling/nonfouling biomaterials. Polymer 2010, 51 (23), 5283-5293. 